\title{
Impairment Constrained Based Routing for Managed Reach WDM Optical Networks
}

\author{
Dimitrios Vogiatzis, Carmen Mas Machuca, and Ioannis Tomkos \\ Athens Information Technology (AIT) Center, Markopoulo Av. PO Box 68, 19002 Peania, \\ Athens, Greece \\ $\{$ dvog, cmas, itom\} @ait.edu.gr
}

\begin{abstract}
Managed reach networks are networks that allow placing of regenerators in the main cities and at requested distances. These networks minimize the number of regenerators but need a routing approach able to establish the connections through regenerating nodes when the signal quality deteriorates. For this purpose, Impairment Constrained Based Routing (ICBR) is used. Considering that impairment related information is exchanged through signaling, the ICBR should be able to compute paths that guarantee a certain level of optical signal quality at the receiver. In this paper we propose an ICBR that is combined with a Routing and Wavelength Assignment algorithm. The proposed ICBR will be presented and used with two different impairments: the Polarization Mode Dispersion (PMD) of the fibers and the Amplified Spontaneous Emission (ASE) noise of the amplifiers. Savings in the number of regenerators is shown when the proposed ICBR is used instead of traditional routing algorithms such as minimum hop and shortest path. Dependence of the blocking probability on different parameters such as span length and $\mathrm{OSNR}_{\min }$ is also demonstrated.
\end{abstract}

\section{Introduction}

Today's optical networks are opaque, i.e. regeneration of all the channels is done at each network node. This solution allows the signal to travel and reach large distances; however it is quite expensive due to the number of regenerators needed in the network and the connection bit-rate dependency. One proposed solution is transparent optical networks [1]. In transparent optical networks the signal is transported end-to-end all optically, without being converted to the electrical domain along its path. Another solution called managed reach networks was recently proposed [2] and is a compromise between transparent and opaque networks. This scenario proposes a network that allows placing of regenerators in the main cities and at requested distances. This approach minimizes the number of regenerators but needs a routing approach able to route the connections through regenerating nodes when the signal quality deteriorates.

Routing in opaque networks assumes optical link engineering on a per link basis, whereas in transparent network is done on a per connection basis [3]. Transparent and managed reach networks require routing approaches that take into account the physical limitations of the optical layer: in the former it will limit the transparency reach, whereas in the latter it will determine the number of regenerators needed. The reason 
is that traditional routing protocols use simple cost parameter (e.g. link length) and the resulting paths do not guarantee that the received optical signal, which has been transparently transported through the network, has enough quality. These physical impairments should be taken into account by the routing protocol. For this purpose, impairment information should be exchanged between the network components through signaling. MPLS and GMPLS platforms are able to support the signaling and the routing required to perform Impairment Constraint Based Routing (ICBR)[4].

In order to deal with this problem, this study has been focused on the analysis of the number of regenerators that can be saved when the routing approach used in the network takes into account the physical impairments.

\section{Routing in Transparent and Managed Reach Networks}

In opaque optical networks the signal is electrically regenerated at each node, i.e. any impairment of the signal can be eliminated. In these networks, the used routing algorithm gives for each connection request a path that may use different wavelengths (electrical conversion is available nowadays). Existing routing approaches find a path minimizing a certain cost parameter such as the number of hops or the fiber length. Routing in transparent and managed reach networks is more complicated due to the absence of optical conversion and regeneration. In this case, routing is in charge of finding a path with a common available wavelength through all the links of the path. The routing algorithm should take into account the physical limitations of the path in order to guarantee that the optical signal will reach the receiver with acceptable quality. This happens because the optical signal in a transparent network cannot be easily monitored and can be easily deteriorated since signal impairments accumulate along the all-optical path. The routing algorithms that take into account the physical impairment are known as Impairment Constrained Based Routing (ICBR).

In transparent and managed reach networks, not only the path but also the wavelength should be determined. This problem is identified as Routing and Wavelength Assignment (RWA). The logical connections between any pair of edge nodes will be established using lightpaths. Previously proposed RWA schemes assume that once the path and the wavelengths have been identified, the connection is feasible to be established. However, in real world networks this assumption is not necessarily true. Certain physical layer optical impairments may prevent the connection establishment through the proposed path because the signal quality will be severely affected.

\section{Impairment Constrained Based Routing and Wavelength Assignment in All-Optical Networks}

The proposed algorithm performs impairment constrained based routing and wavelength assignment taking into account defined physical constraints. In this work we will present how the algorithm takes into account the PMD and the ASE constraints.

The inputs of the algorithm are:

- End-to-end connection requests: The connection requests, given in Gbps represent the traffic between two cities. 
- Physical layer parameters: Several parameters related with the physical layer characteristics should be given such as the optical fiber attenuation, gain of the amplifiers, bit rate, launched optical power, noise figure of the amplifiers, $\mathrm{OSNR}_{\min }$, span and link lengths.

The output of the algorithm is for a given connection request, a path and an assigned wavelength, whenever the connection is not blocked.

Initially (preprocessing phase), the source-destination nodes of all the connection requests are identified and the bit rates of the connections are specified, based on traffic models that predict the traffic increase on a year per year basis. Additionally, the link costs are computed based on the physical layer and the 'cost impairment' parameters which are related to the impairments that should be taken into account when finding the paths. When ASE is under consideration, either on its own or in combination with the PMD, the cost parameter is the number of amplifiers per link, whereas when only the PMD constraint is examined, the corresponding cost parameter is the $\mathrm{D}_{\mathrm{PMD}}$ of the link along with the link length. Once the link costs are found, the routing and wavelength assignment (RWA phase) may begin. For this purpose, $k$ shortest paths for each source-destination pair are computed. The $k$ parameter is set before running the algorithm and in our simulations $k$ has been set to 3 . These $k$ candidate paths are used for the RWA problem. Since the only parameter that captures the real performance of a network at the end of the day is the blocking probability, we have tried to reduce the blocking probability by evenly distributing the traffic in the network, so that load balancing is achieved. For this reason, the algorithm that we have used for the RWA problem attempts to minimize the cost induced by all flows in the links of the network, by a proper selection of a monotonically increasing function, as a flow cost function. The RWA module [6] selects the 'best' paths with the available wavelengths, which guarantee minimization of the blocking probability. This module constantly interacts with the Impairment Aware Constrained Based Routing module. Starting with the shortest path delivered from the RWA module, and continuing with the others, it is checked whether the path accomplishes the considered impairment requirements. If so, the connection is established; otherwise and if none of the paths delivered from the RWA module satisfies the physical impairment requirements, the connection is blocked.

Some of the novelties of the proposed algorithm are:

- It is suited for all types of topologies. It has been shown that the RWA problem can be partitioned into two sub-problems: the routing and the wavelength assignment. However, this partitioning is possible with star and ring topologies but not in mesh networks [4]. Our approach merges both sub-problems and is able to find optimal solution in mesh networks.

- It is also well known [5], that dividing the RWA problem into two distinct subproblems, it yields only to sub-optimal results. Optimality can be only achieved, if the two sub-problems are treated as one joint optimization problem. Our algorithm guarantees this, by the proper selection of the k-shortest paths, which give a good representation of the space where the optimal solution lies in.

- The algorithm is recursive, that is, if the first solution does not accomplish the impairment constrains, a second solution may be found. 


\section{Simulation Scenario}

As basis for our simulations we used the pan-European network shown in Fig. 1. This network consists of 8 nodes interconnected by 16 bidirectional links. The cumulative distribution of the fibre link lengths between the nodes is shown in Fig. 2. The average link length is about $700 \mathrm{~km}$ and each link is able to support up to 80 wavelengths. The fibers that are considered can be either 'new' (PMD factor equal to $0.1 \mathrm{ps} / \sqrt{\mathrm{km}}$ ) or 'old' (PMD factor equal to $0.5 \mathrm{ps} / \mathrm{km}$ ). The parameter $a$ gives the percentage of new fibers in the network.

The considered traffic matrix is static and consists of 56 end-to-end connections (full connectivity). The connections matrix has been considered to be symmetric and with values of 10,20 and $40 \mathrm{Gbps}$ bit rates (i.e. each connection between a pair of cities will be mapped into a single wavelength of the abovementioned capacity).

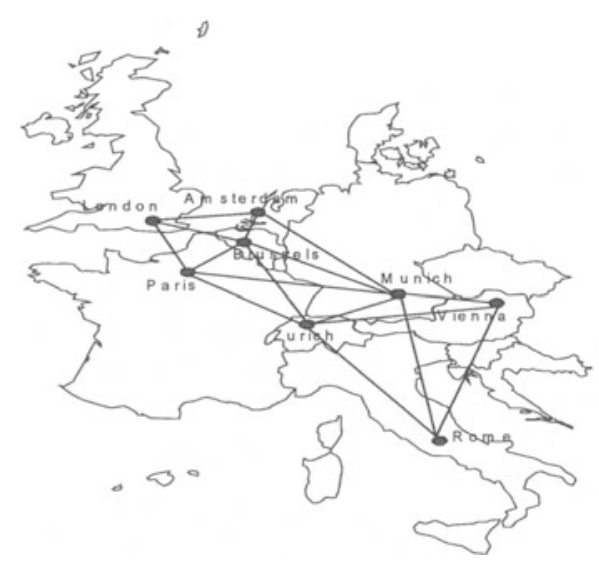

Fig. 1. Topology of the reference network

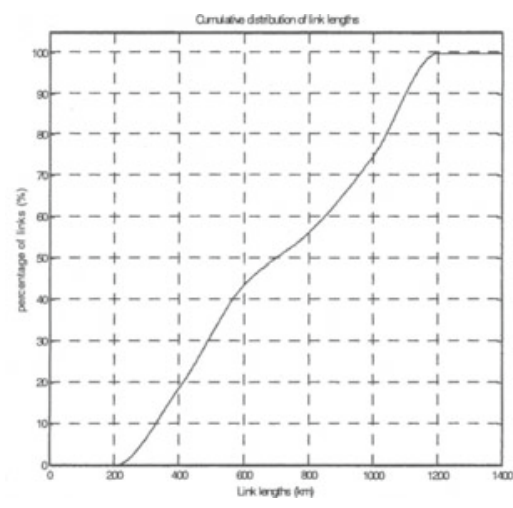

Fig. 2. Link length cumulative percentage

\section{Simulation Results}

\subsection{ICBR Studies Considering the PMD Impairment as a Constraint}

In this scenario connections are routed with ICBR taking into account the PMD parameter of the fibers. As mentioned earlier, our study focuses on managed reach networks, which are networks that locate regeneration where is needed due to the effect of impairments. In particular, this study has looked at the number of regenerators that are required when different routing algorithms are used. These algorithms are: the minimum hop, the shortest length and the proposed Impairment Constrained Based Routing (ICBR). The number of regenerators required in the $10 \mathrm{Gbps}$ connections routed by the different routing schemes for different percentage of new fibers has been plotted in the graph of Fig. 3. This graph clearly shows that when the percentage of new fibers (x-axis) increases, the number of regenerators (y-axis) needed when using ICBR decreases much faster than with the other two routing algorithms. The 
percentage of fewer regenerators needed with ICBR compared to minimum hop increases as the percentage of new fibers increases. For example for $a=0.4$, ICBR requests $75 \%$ less regenerators than minimum hop and for $a=0.6$ ICBR requests $90 \%$ less regenerators than minimum hop. In the particular case when the percentage of new fibers is 0.8 , no regeneration is needed if ICBR is used.

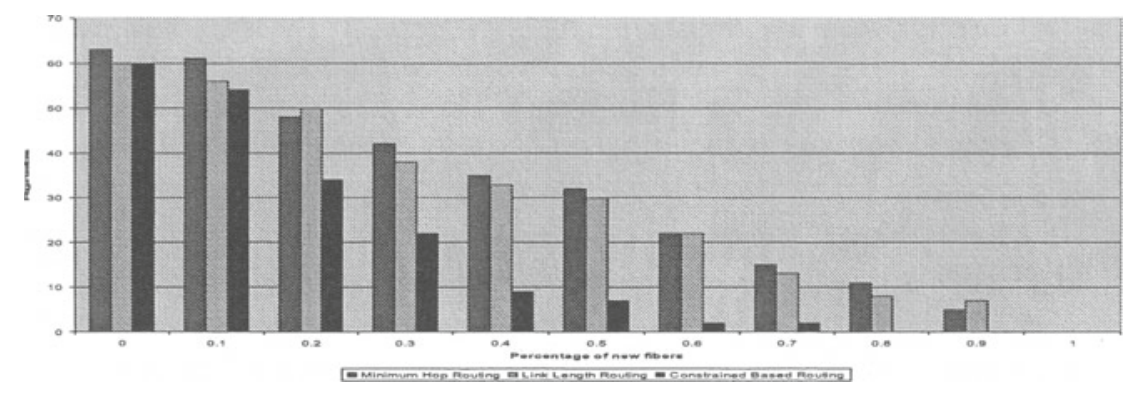

Fig. 3. Number of regenerators vs. the percentage of new fibers for $B=10 \mathrm{Gbps}$

\subsection{ICBR Studies Considering the ASE Impairment as a Constraint}

The connections are now routed based on the ASE noise. In the simulations a homogeneous network has been considered and therefore, the results of minimum hop and shortest length routing, which are the same have been compared with the proposed ICBR. In case of non-homogeneity, the results of minimum hop and shortest length will differ but still ICBR uses fewer resources and outperforms the other two.

Let us consider the core network shown in Fig. 1 to be transparent. This section is focused on the study of the blocking probability versus the span length in function of the bit rate and the OSNR ${ }_{\min }$. Different OSNR $_{\min }$ have been considered (each of them related to different FEC schemes). Fig. 4 shows the increase of the blocking probability with the $\mathrm{OSNR}_{\min }$ value and also with the bit rate. The blocking probability also increases for longer span lengths since although there will be fewer amplifiers, the cumulative noise will be higher and therefore more connections will not be able to accomplish the minimum OSNR required and will be blocked.

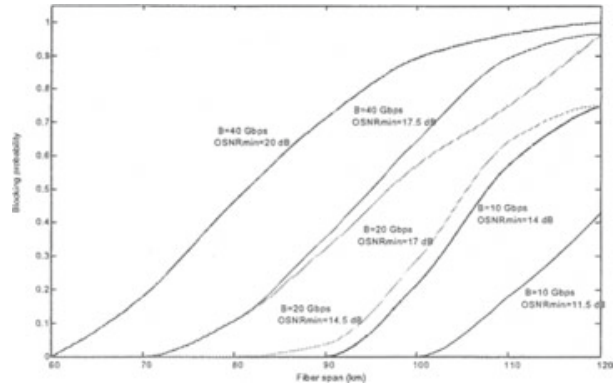

Fig. 4. Blocking probability vs. fiber span length wrt. OSNR ${ }_{\min }$ and connection bit rates

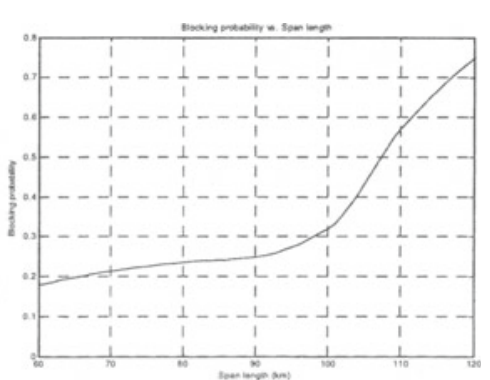

Fig. 5. Blocking probability vs. span length taking into account PMD and ASE 


\subsection{ICBR Studies Considering the PMD and ASE Impairments as Constraint}

The two constraints have been considered as parameters of our proposed ICBR. In our case, we looked at the scenario of span lengths of $70 \mathrm{~km}$, bit rates of $10 \mathrm{Gbps}$, PMD parameter equal to $0.25 \mathrm{ps} / \mathrm{km}$. The blocking probability versus the span length has been plotted in Fig. 5. This figure shows that there is a smooth increase of the blocking probability for span lengths shorter than $90 \mathrm{~km}$ and that after this value, the blocking probability increases much abruptly. This is due to the fact that for spans shorter than $90 \mathrm{~km}$, the dominant impairment is the PMD, whereas for longer spans, both impairments contribute to the increase of blocking probability.

\section{Conclusions}

This paper described the most important issues related to transparent and managed reach optical networks. It has been shown that in these networks Impairment Constrained Based Routing should be used to guarantee that, by the proper selection of the paths, the quality of received optical signal is within acceptable limits. The presented results take into account as impairment constraints the fiber PMD and the cumulative ASE noise. One of the results is the reduction in the required number of regenerators when the proposed ICBR is used instead of the tradition minimum hop and shortest length routing algorithms. For example, when considering the PMD as constraint, the number of regenerators that could be saved increases with the percentage of new fiber installed in the network, since ICBR tries to route the connections through more new links. In some scenarios the number of regenerators needed with ICBR is null. Other results study the blocking probability and its dependency based on different parameters such as span length, percentage of new fibers, or OSNR min $^{\text {. In }}$ the conference, details on the algorithm and the simulations will be presented.

\section{References}

1. R. E. Wagner et al., 'MONET: Multiwavelength optical networking', Journal of Lightwave Technology, 14(6), 1349-1355.(1996).

2. R. Wagner "Evolution of Optical Networking", LEOS 2000 Proceedings, TuC1, Puerto Rico, November 2000.

3. Frank Chang and Mazda Salmanian "Routing requirements in photonic networks" National Fiber Optic Engineers Conference (NFOEC), Orlando, September 2003.

4. A.Chiu, J. Strand and R. Tkach "Unique features and requirements for the optical layer control plane" IETF draft, May 2001.

5. John Strand, Angela L. Chiu, and Robert Tkach "Issues for routing in the optical layer" IEEE Communications Magazine, February 2001, pp.81-87.

6. Dimitrios Vogiatzis "Routing and Wavelength Assignment in WDM Optical Networks" Diploma thesis, Computer Engineering and Informatics Department, University of Patras, June 2002. 